\title{
STUDIES ON THE EFFECT OF CARDIAC IRREGULARITY ON THE CIRCULATION
}

\author{
II. The Estmation of Cardiac Output in Dogs Subject to \\ Artificial Auricular Fibrillation
}

By HAROLD J. STEWART AND A. R. GILCHRIST

(From the Hospital of the Rockefeller Institute for Medical Research, New York)

(Received for publication July 8, 1927)

In preceding papers $(1,2,3)$ we have reported observations upon the rate of blood flow in regular and irregular tachycardias in normal unanesthetized dogs. In those experiments the values for the relative blood flow were obtained by calculating the ratio of the oxygen removed from each liter of blood by the tissues during the artificial rhythm to the oxygen removed during the normal rhythm, the ratio during the normal period being placed at 100 . We did not estimate the oxygen consumption because of the reaction of untrained unanesthetized animals to the procedures used in measuring it. We did not wish to anesthetize the animals because of the secondary changes that accompany anesthesia. Because of the undesirable effect of morphine on the respiration and in slowing the heart rate we decided against its use.

We have also performed a few experiments on trained unanesthetized dogs. In these we have been able to measure the oxygen consumption and calculate the minute volume output of the heart (Fick's method). The results in these experiments agreed so closely with the calculations made of the relative blood flow that it seemed useless to accumulate a larger amount of data on the minute volume output of the heart under these conditions than is here presented.

\section{METHODS}

All the dogs were trained to breathe in a Benedict spirometer before they were used in these experiments for 20 to 30 minutes a day for 10 days to 2 weeks. At the end of this time they lay quietly on the board during metabolism tests without 
emotional disturbance. It was possible to decide at the first or second trial whether a given dog was apt to be satisfactory and we continued to train only those showing evidence of being adaptable. The rubber mask described by Blalock was used and found to be satisfactory (4).

After the dogs were sufficiently trained they were operated on in order to sew wire electrodes to the right auricles as previously described (1). Twenty-four hours after this preliminary operation the heart was stimulated through these electrodes and the effect of the induced rhythm on the cardiac output studied. The dogs were in a basal metabolic state on the day of the experiment and lay quietly on the board without anesthesia. Samples of arterial blood were drawn from a femoral artery and those of mixed venous blood from the right ventricle by means of a special cannula inserted in to that chamber through the right external jugular vein (5). The oxygen content of these samples was estimated by the Van Slyke and Neill manometric method (6). The difference between the oxygen content of the arterial and of the mixed venous blood gives the amount of oxygen removed from the blood by the tissues or, from the point of view of the lesser circulation, the amount of oxygen taken up by the blood in passing through the lungs. Immediately after the blood samples were drawn, the oxygen consumption was measured with a Benedict spirometer equipped with a graphic recording drum.

Data were therefore at hand for calculating the minute volume output of the heart according to the principle of Fick (7):

Cubic centimeters of oxygen consumed per minute $=$ Cubic centimeters of blood passing Amount of oxygen absorbed by 1 cc. of blood in
passing through the lungs

Estimations of the cardiac output were made during the normal control period and again 60 minutes after the onset of artificial fibrillation of the auricles and while this was still present. Flectrocardiograms and simultaneous pulse tracings were taken at the time the cardiac output was measured in order to be certain of the rhythm then present as well as to calculate the ventricular rate and pulse deficit (3).

In 4 dogs we have measured the volume output of the heart during the normal rhythm and during auricular fibrillation. In one dog we have observations as well during regular tachycardia. As before, faradic current was used to bring on auricular fibrillation (1) and rapidly repeated single induced break shocks to institute regular tachycardia (2).

\section{OBSERVATIONS}

The effect of auricular fibrillation on the oxygen consumption. The oxygen consumption remained constant throughout these experiments. The change varied between a 2 per cent increase and an 8 per cent decrease, only one $\operatorname{dog}(\operatorname{dog} C)$ showing a change of this magnitude 
HAROLD J. STEWART AND A. R. GILCHRIST

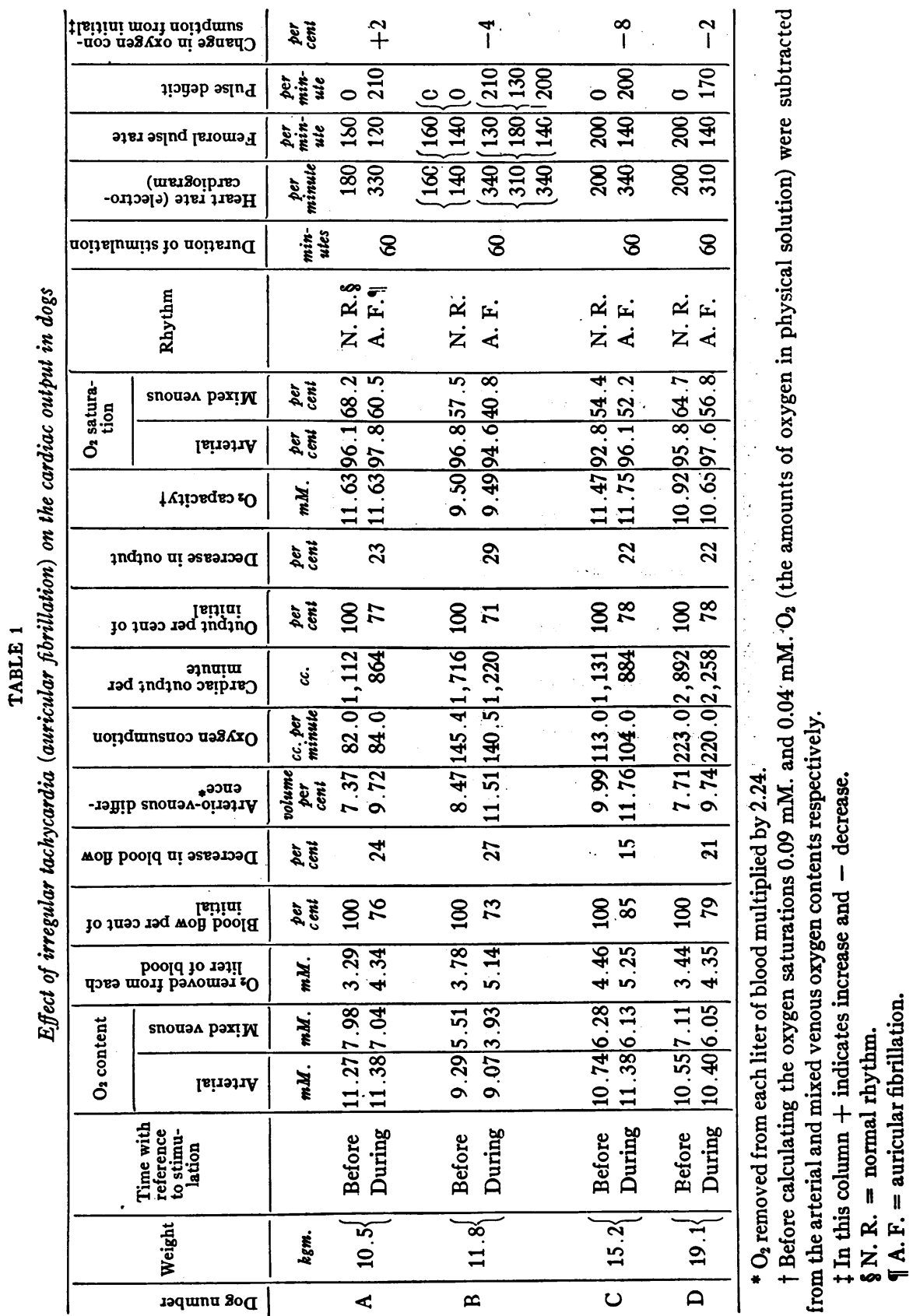




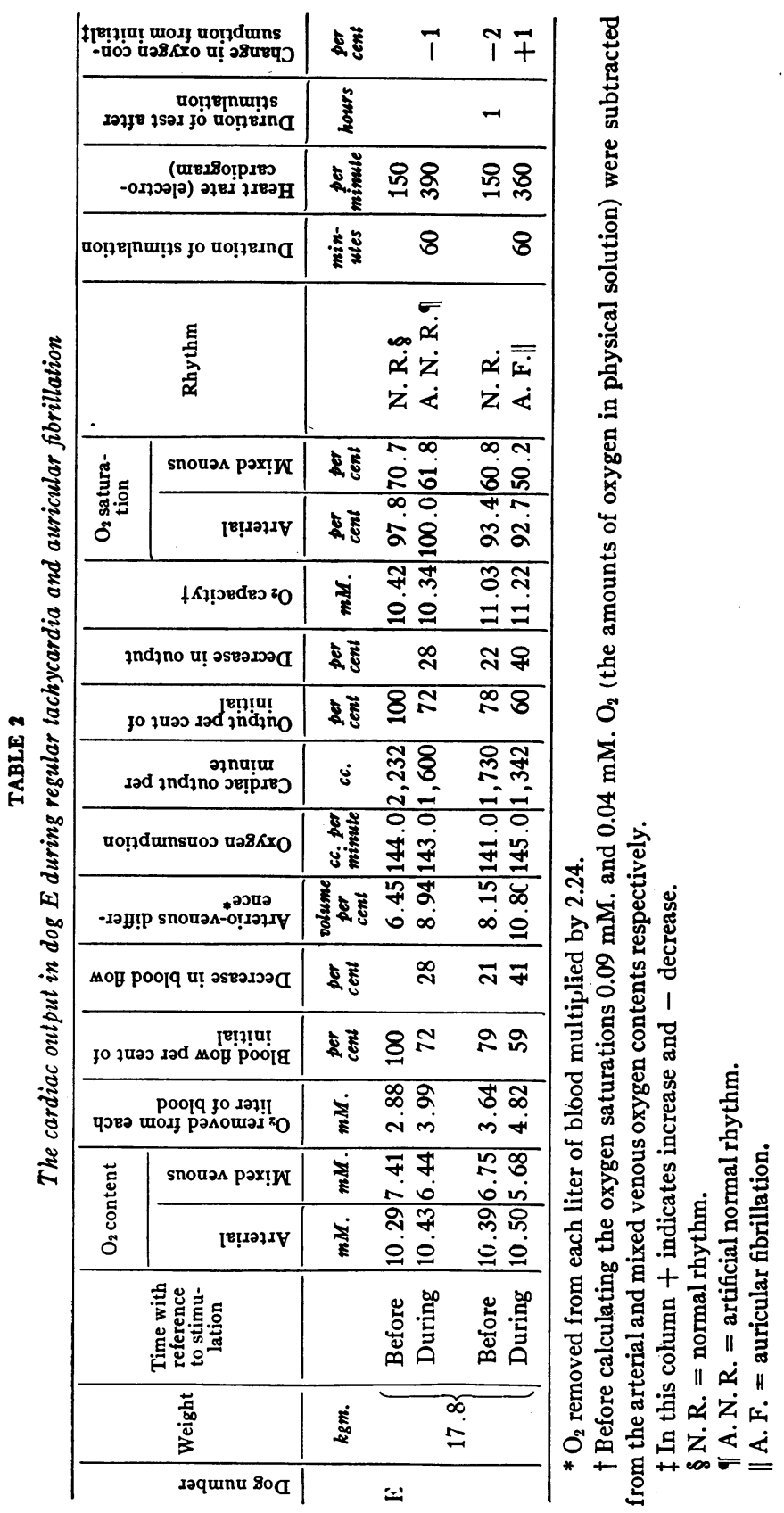




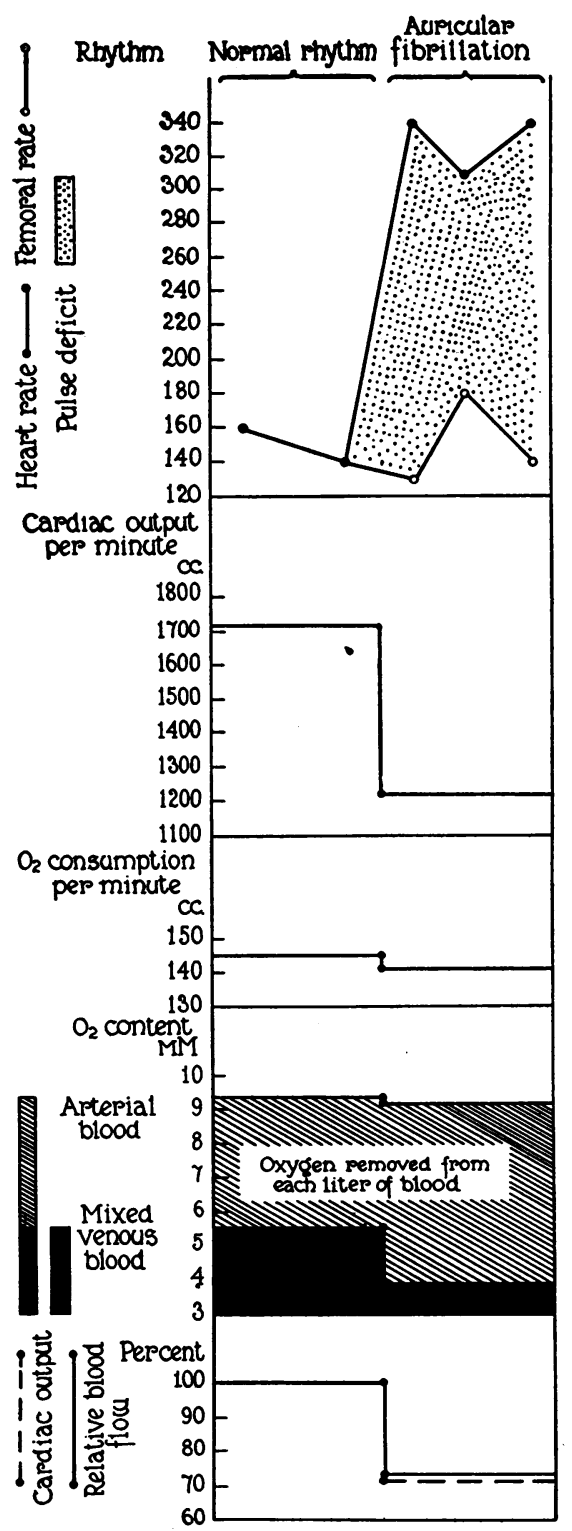

Fig. 1. The Effect of Auricular Fibrillation upon the Cardiac Odtput AND OXYgen Consumption in Dog B Is INDiCATED

Graphitcally in This Figure

The change in cardiac output is compared with the relative blood flow. During the normal rhythm they are both represented by 100 per cent and the lines coincide. During auricular fibrillation the cardiac output is decreased to 71 per cent of its initial value, while the relative blood flow fell to 73 per cent of its initial flow. The difference between these two is only 2 per cent. 
(table 1). These variations are well within the limit of error involved in this method of measuring the oxygen consumption.

The effect of auricular fibrillation on the cardiac output per minute. There are observations on 4 dogs. In all there was a decrease in the cardiac output per minute below the level found during the normal rhythm. The decrease varied between 22 and 29 per cent of the initial output (table 1). The ventricular rates during the normal rhythm ranged from 160 to 200 per minute and during auricular fibrillation between 310 and 340. During the normal rhythm a pulse deficit was not present, while during auricular fibrillation it varied between 130 and 210 per minute.

Comparison of the effect of auricular fibrillation and regular tachycardia on the cardiac output

In $\operatorname{dog} \mathrm{E}$ (table 2) the cardiac output was measured during regular tachycardia as well as during auricular fibrillation. The oxygen consumption remained constant; there was a 2 per cent decrease and 1 per cent increase. The cardiac output in this dog decreased 28 per cent during regular tachycardia of 390 per minute. After a short rest period the cardiac output increased slightly toward normal. Then, when auricular fibrillation was instituted, the cardiac output decreased to 40 per cent below the initial value. This dog belongs to the small group of dogs in which the rate of blood flow was found to decrease during regular tachycardia (2).

A comparison of the changes in the relative blood flow and the cardiac output during auricular fibrillation and regular tachycardia

There is close agreement between the changes in relative blood flow and the percentage changes in the minute volume output of the heart in these experiments. In $\operatorname{dog} B$ the cardiac output was decreased from $1716 \mathrm{cc}$. per minute during the normal rhythm to $1220 \mathrm{cc}$. per minute during rapid auricular fibrillation (fig. 1); that is to say it fell to only 71 per cent of the normal output. The relative blood flow during the period of auricular fibrillation was 72 per cent of the flow during that of the normal rhythm. There was agreement, therefore, between the two measurements, the cardiac output and relative blood flow to 
within 2 per cent. The decreases in the relative blood flow in these dogs ranged from 15 to 41 per cent; the decreases in cardiac output, from 22 to 40 per cent (tables 1 and 2). For any one dog the two agree within 2 per cent in all instances except one; in this one $(\operatorname{dog} C)$ the difference was 7 per cent. This is within the limit of error of the methods for only changes greater than 10 per cent have been considered significant.

\section{DISCUSSION}

The measurements of relative blood flow in these experiments agree with the changes in cardiac output whenever this was actually measured. The estimations of the relative blood flow were based on the assumption that the metabolism of the dogs (oxygen consumption) remained constant during the period of the experiments. We put this to test; we measured the oxygen consumption in these animals during the artificial rhythms and found that it remained constant. The assumptions made in the preceding papers (1,2 and 3), therefore, that metabolism remained constant and that the changes which we observed were due to changes in rate of blood flow and not to changes in tissue metabolism, were therefore correct, and our inferences based on these assumptions are probably valid.

From these experiments we gain no further insight into the mechanism which is responsible for the decreased cardiac output or decreased rate of blood flow in auricular fibrillation. This subject has been discussed in another paper (3).

\section{SUMMARY}

We have measured the cardiac output of normal unanesthetized dogs during auricular fibrillation and during regular tachycardia and found that:

1. Under the conditions of these experiments the oxygen consumption of the dogs remains constant. This is true whether the natural normal rhythm is present or whether it is that of artificial auricular fibrillation or regular tachycardia.

2. The cardiac output of the heart per minute is less during rapid auricular fibrillation than it is during the normal slower rhythm. 
3. The estimations of the relative blood flow were found to run parallel with the changes in cardiac ouput measured by the method of Fick during artificial auricular fibrillation and regular tachycardia as well as during the natural normal rhythm.

\section{CONCLUSIONS}

1. The heart is less effective in the propulsion of blood during rapid auricular fibrillation than it is during the normal slower rhythm.

2. Since the oxygen consumption remains constant in these experiments, calculations of the relative blood flow in experiments carried out in a similar manner are valid and the conclusions at which we have previously arrived from such data are valid.

\section{BIBLIOGRAPHY}

1. Stewart, H. J., Crawford, J. Hamilton, and Hastings, A. B., J. Clin. Invest., 1926, iii, 435. The Effect of Tachycardia on the Blood Flow in Dogs. I. The Effect of Rapid Irregular Rhythms as Seen in Auricular Fibrillation.

2. Stewart, H. J., and Crawford, J. Hamiton, J. Clin. Invest., 1926, iii, 449. The Effect of Tachycardia on the Blood Flow in Dogs. II. The Effect of Rapid Regular Rhythm.

3. Stewart, H. J., Crawford, J. Hamilton, and Gilchrist, A. R., J. Clin. Invest., 1928, v, 317. Studies of the Effect of Cardiac Irregularity on the Circulation. I. The Relation of Pulse Deficit to Rate of Blood Flow in Dogs Subject to Artificial Auricular Fibrillation and to Regular Tachycardia.

4. Blalock, A., J. Lab. and Clin. Med., 1927, xii, 378. A Rubber Mask for Determination of Oxygen Consumption.

5. Stewart, H. J., J. Biol. Chem., 1925, lxii, 641. The Oxygen and Carbon Dioxide Contents of the Arterial and Mixed Venous Blood in Normal Intact Dogs.

6. Van Slyke, D. D., and Neill, J. M., J. Biol. Chem., 1924, Ixi, 523. The Determination of Gases in Blood and Other Solutions by Vacuum Extraction and Manometric Measurement. I.

7. Fick, A., Verhandl. d. Würzb. physikal. -med. Gesellsch. 1870. N. F. ii. S. xvi. der Sitzungsber. Ueber die Messung des Blutquantums in der Herzventrikeln. 